\title{
Le roman comme une possibilité d'une expérience fantasmée
}

Claudine GAETZI

Viceversa littérature

\begin{abstract}
Pascale Kramer considère le roman comme un modèle expérimental qui lui permet de répondre à des interrogations existentielles. Dans Le Bateau sec (1997) et L'Implacable Brutalité du réveil (2017), elle se focalise sur le personnage d'une mère confrontée à des difficultés dans sa relation avec sa fille. Dans les deux romans apparaît un écart troublant entre le savoir de la voix narrative et le savoir des personnages. Le personnage semble tantôt ne pas saisir ce que la voix narrative analyse avec précision, tantôt capable d'exprimer ce qu'il ressent d'une manière quasi autonome. Le roman peut-il répondre aux questions qui préoccupent Pascale Kramer ? La fiction n'aurait-elle pas plutôt pour fonction de nous faire vivre des affects fantasmatiques, tout en laissant les interrogations ouvertes?
\end{abstract}

Keywords : Pascale Kramer, fiction, modèle expérimental, interrogations, maternité, affects

Lorsqu'on lit des interviews de Pascale Kramer, il apparaît qu'elle considère le roman comme la possibilité de mettre à l'épreuve, au moyen de l'écriture, sur un mode expérimental, les sentiments, les émotions, les doutes, les difficultés et les questionnements auxquels on peut se trouver confronté dans la vie, et cela sans les avoir soi-même vécus, en se projetant dans l'imaginaire des personnages qu'elle crée.

Au sujet de L'Implacable Brutalité du réveil (2017) Pascale Kramer explique qu'en rendant visite à une amie qui venait d'accoucher, elle a été saisie par la dimension inéluctable de la situation : « Lorsque je l'ai vue près de la piscine avec son bébé dans les bras, soudain je me suis dit : que fait-on lorsqu'on découvre qu'être mère est une erreur et que l'on n'assume pas ce rôle? » (Rousseau 2009)

L'écrivaine s'est projetée dans cette relation mère-enfant, en se demandant comment elle-même réagirait : « Mais qu'est-ce qu'on fait si tout d'un coup on réalise que mettre au monde est un choix irréversible et qu'on a peut-être fait une erreur ? J'avais l'impression de vivre à la fois mon amour des enfants et la crainte de ne pas être à la hauteur d'un devoir maternel » (Adamo 2019).

Dans L'Implacable Brutalité du réveil, elle porte ce questionnement en se focalisant sur le personnage d'Alissa qui vient d'accoucher : "Je me suis mise d'abord dans la tête d'une mère » (Rousseau 2009). 
Toutefois, même si Pascale Kramer prend le parti d'entrer dans un imaginaire maternel, elle rédigera son roman à la troisième personne, comme s'il était pour elle nécessaire de se tenir à une certaine distance de son personnage. Plutôt que de recourir au je et de s'immerger totalement dans le rôle, elle observe ce qui se passe, tout en se réservant un accès privilégié à l'intériorité du personnage sur lequel elle se focalise. Ainsi, quand Alissa, au bord de la piscine, allaite Una, on apprend ce que la mère ressent physiquement : «Alissa se concentra sur le mâchonnement des gencives dont la sensation la troublait» (2009: 9).

Le Bateau sec (1997) et L'Implacable Brutalité du réveil (2009, 2017'), à une dizaine d'années d'intervalle, explorent spécifiquement les aléas de la relation mère-fille. Dans cet article, il s'agira d'examiner comment l'écrivain construit une narration qui lui permet, et qui permet au lecteur, de vivre par procuration des situations complexes et intimement perturbantes. Dans ces deux romans, une focalisation interne permet d'accéder aux pensées et sentiments de la mère. Le récit des événements est suivi, assez systématiquement, d'une analyse du ressenti de Suzan dans Le Bateau sec, et de celui d'Alissa dans L'Implacable Brutalité du réveil.

La voix narrative s'attache à décrire minutieusement ce qu'éprouvent les mères dans ces deux romans, mais il apparaît qu'un certain nombre d'éléments demeure sans explication pour les personnages. Ce décalage du savoir entre la voix narrative et les personnages incite à s'interroger sur la manière dont un roman peut, ou non, aider l'écrivain et son lecteur à répondre à des questions existentielles et à appréhender des situations réelles.

\section{Une analyse psychologique qui se jouerait à l'insu des per- sonnages?}

\section{Le Bateau sec : imperfection et impuissance de l'amour maternel}

Dès le début du roman, on apprend que le caractère d'Ann, jeune adolescente capricieuse et menteuse, inspire de l'inquiétude à sa mère : " elle était habile et lassante, d'une versatilité que Suzan craignait parce qu'elle n'arrivait pas tout à fait à en évaluer les dangers » (Kramer I997 : I3). Suzan et sa fille, Ann, âgée de dix-sept ans, se rendent dans un hôtel qui ressemble à " un grand bateau sec échoué » (I3); il est tenu par Tom, un ami de la mère. Suzan souhaitait venir s'y reposer durant trois semaines, seule, mais Ann, grâce à l'appui de son père, a obtenu de l'accompagner, ce dont Suzan garde « un sentiment de rancune, cruel parce que tellement inutile» (I5).

I Les numéros de pages indiqués pour les citations de L'Implacable Brutalité du réveil correspondent à la réédition de 2017. 
L'origine de l'amitié qui existe depuis des années entre Tom et Suzan est très intime : «Suzan avait été très proche de lui, au début de son mariage, au point de lui confier ce qu'elle s'avouait à peine : le malaise où l'avait plongée sa grossesse, un état de presque anesthésie contre lequel elle se sentait désarmée » (28-29). Dès le début de sa maternité, Suzan apparaît dépassée par ce qui lui arrive. On ne sait pas comment se déroule l'enfance d'Ann, mais à l'adolescence, et en particulier lors de ce séjour à l'hôtel, la relation mèrefille est extrêmement compliquée, comme si Suzan était sans cesse victime d'une situation qu'elle n'a pas souhaitée, mais à laquelle elle ne sait pas s'opposer : elle voudrait être seule, mais Ann lui impose sa présence, de manière intempestive, tantôt boudant, tantôt réclamant tendresse et réconfort. Suzan observe sa fille, dont la conduite la choque souvent par son égocentrisme et son absence d'égards pour autrui, avec une étrange fascination et des espoirs secrets qu'elle ne voit pas se réaliser :

Suzan la dévisagea un instant, animée par cette curiosité parfois fière, toujours étonnée, qu'elle n'avait jamais pu identifier comme une véritable tendresse de mère, se mettant très tôt en porte-à-faux avec une maternité dont les bonheurs se faisaient encore attendre (30).

Le fait que Suzan laisse Ann, qui n'a évidemment pas son permis, conduire sa voiture, indique dès les premières pages que la mère accepte que sa fille, au mépris des lois, soit aux commandes et les mette en danger en roulant vite. Un programme est ainsi esquissé, où l'on comprend que la mère se plie aux volontés de sa fille, et ne sait pas se protéger d'elle. À l'hôtel, Suzan a de la peine à supporter la présence d'Ann, ses sautes d'humeur, ses caprices, mais quand celle-ci s'éloigne durant quelques jours avec Grégoire, le fils de Tom, cela ne la soulage pas : «Suzan entendit retomber le silence sur une solitude qu'elle avait tant espérée mais qu'Ann avait sciemment, savamment saccagée avant de s'en aller » (59). Suzan ne parvient pas à profiter de cette trêve, elle tombe malade, et lorsque sa fille revient et se comporte en tyran, Suzan ne proteste jamais ouvertement, tout en étant très irritée. La mère apparaît constamment et profondément ambivalente, cédant à contrecœur aux exigences de sa fille, la câlinant lorsqu'elle le réclame, mais sans affection, l'observant avec agacement, voire avec consternation, et parfois avec admiration. Elle ne la réprimande jamais, comme prise par une fascination qui la dépasse et dont elle espère en vain l'accomplissement.

La construction narrative du Bateau sec ressemble à un mécanisme dont chacun des éléments mène à un drame que l'on redoute dès les premières pages, la voix narrative nous ayant averti que Suzan était incapable d'évaluer les dangers que le comportement de sa fille était susceptible de provoquer. Au bout de quelques jours de ce séjour à l'hôtel, survient alors «le 
premier événement vraiment irréparable de ces vacances » (76). Suzan, depuis la terrasse de l'hôtel, a le regard qui plonge involontairement sur la chambre où Ann et Grégoire font l'amour; Ann s'aperçoit que sa mère les a observés. Le lendemain, Ann prend la voiture et disparaît. Mais cette fugue sera de courte durée. À son retour, Suzan décide d'écourter leur séjour, mais Ann réussit à l'en dissuader, affirmant qu'elle est amoureuse de Grégoire, ce qui incite le jeune homme à annoncer « qu'il restait lui aussi encore un peu » (II4). La voix narrative se fait alors annonciatrice d'un dénouement tragique : "Suzan réalisa plus tard que le drame s'était joué ce jour-là par ces mots, mais sans doute était-il déjà trop tard pour bien faire » (II4). Ce fatalisme résonne étrangement en écho avec l'angoisse ressentie par Pascale Kramer à l'idée que mettre un enfant au monde est un choix irréversible, comme une sorte d'engrenage inéluctable, sans retour possible. Dans Le Bateau sec, les agacements et mécontentements réciproques s'additionnent, jusqu'à faire basculer inexorablement un quotidien somme toute assez banal dans la tragédie.

Cela commence par une vengeance : Ann, déstabilisée par la désapprobation générale que sa fugue a suscitée, et ulcérée par l'attitude désormais distante de Grégoire, s'en prend à l'oiseau handicapé par une aile brisée qui a trouvé refuge sur la terrasse de l'hôtel. Elle le blesse cruellement et appelle sa mère pour lui montrer l'oiseau prostré par la souffrance : il a le bec arraché. Elle convainc sa mère de le tuer. Suzan s'exécute. Elle lui écrase le crâne et le laisse lentement agoniser. Elle pense que sa fille est coupable d'avoir mutilé l'oiseau, mais elle ne lui reproche rien, ni ne l'incite à avouer son méfait : «Il était de plus en plus certain qu'[Ann] avait voulu tuer l'oiseau imaginant sans doute par cet acte faire triompher enfin son malheur - mais qu'elle n'avouerait jamais le crime dont la lenteur l'avait comme épuisée » (I48). Elle la laisse ensuite seule dans sa chambre, "se sentant pour une fois et pour de bon, la liberté de ne pas chercher à la comprendre » (I49). Cet acte malveillant fait perdre à Ann le peu de sympathie que Tom et ses enfants, attachés à l'oiseau, éprouvaient encore pour l'adolescente. Ann téléphone alors à son père, qui l'aime éperdument et se montre habituellement très indulgent, mais qui cette fois se fâche. Ann veut rentrer, sa mère refuse froidement. Elles resteront encore deux jours, décide-t-elle, faisant soudain preuve d'une autorité à laquelle sa fille n'est pas du tout habituée.

Le dernier soir, Suzan décide de ne pas emmener Ann au dîner en ville. Lorsqu'elle la laisse seule à l'hôtel et qu'elle la voit « si belle, si sincère » (I62), elle éprouve avec intensité tout ce que son amour a d'irrémédiable : «Elle comprenait enfin qu'on se dépassionne d'un mari, mais jamais d'une fille, même quand l'amour est imparfait, et tellement infructueux » (I62).

Au retour, à cent mètres de l'hôtel, la voiture de Tom, dans laquelle se trouvent ses enfants et Suzan, entre brutalement en collision avec la voiture 
de Suzan, « qu'Ann [au volant] avait garée en plein virage » (I65). Ann meurt sous leurs yeux. « Ni Suzan ni Tom n'osaient sortir de la voiture, horrifiés moins par l'accident lui-même que par l'ampleur de leur culpabilité » (I67). Le lendemain, le père d'Ann arrive et s'efforce de comprendre pourquoi sa fille avait été laissée seule à l'hôtel, mais c'est apparemment inutile : «Suzan se tenait assise devant lui, les mains croisées sur ses genoux, sans pouvoir répondre ni rien justifier, car il n'y avait pas d'explication à tout cela » (I69).

Paradoxalement, le roman se termine en présentant le drame qui a eu lieu comme absurde, inexplicable, alors qu'en tant que lecteur on a assisté au fil des pages à sa minutieuse mise en place. La voix narrative possède le pouvoir de nous faire saisir par quelle logique les personnages sont mus, mais, à eux-mêmes, cette logique échappe.

Douze ans plus tard, lorsque Pascale Kramer entreprend de sonder à nouveau une relation mère-enfant, retrouve-t-on cet écart assez troublant entre le savoir de la voix narrative et le savoir des personnages?

\section{L'Implacable Brutalité du réveil : entre désarroi et lucidité}

Au bord d'une piscine, Alissa allaite son bébé, Una, et elle est saisie par la soudaine " conscience du rien qu'était encore cette vie dont elle avait désormais la charge » (Kramer 2017 : IO). La responsabilité qu'elle doit porter en tant que jeune mère lui paraît écrasante, et elle se sent abandonnée par ses proches, délaissée, dans une solitude à laquelle elle ne sait pas faire face. Elle vient de quitter la maison familiale. Son mari, Richard, et elle ne sont pas encore vraiment installés dans leur nouvel appartement. Sa mère vient la trouver pour lui annoncer qu'elle est amoureuse et que, maintenant que sa fille a quitté la maison familiale, elle divorce et s'en va vivre seule. Alissa ressent comme une injustice et une violence le fait que sa mère se préoccupe désormais de son propre bonheur. C'est un tournant dans leur relation, car jusqu'alors la mère « cédait toujours aux désirs d'Alissa qu'elle devinait avec une formidable intuition, mais cela aussi allait changer » (2I).

Dans ce roman, ce sont donc deux relations mère-fille qui sont analysées avec minutie. En devenant mère, Alissa cesse d'être fille, souffre de l'attitude de sa mère qui la délaisse pour son amant et passe en coup de vent embrasser sa petite-fille, sans guère se préoccuper du désarroi de sa fille. Alissa est incapable d'exprimer ce qu'elle ressent : «Qu'aurait-elle pu lui dire? Que son bonheur l'oppressait? Qu'il la dépossédait de sa propre enfance? » (35).

Alissa se demande si elle a fait les bons choix en épousant Richard et en ayant un bébé. Elle revoit ses ex-collègues de bureau et se sent incapable de reprendre le travail, les difficultés de la maternité l'ayant privée de toute confiance en ses compétences. Alissa apprend que Jim, le mari d'Audrey, va être rapatrié, gravement mutilé pendant la guerre. Être jeune père de famille a évité à Richard de partir combattre, et il se sent coupable de lâcheté. 
On se rend compte que pour les deux parents, la naissance d'Una entraîne des regrets. Leurs rêves et la réalité ne coïncident guère.

Una tombe malade, doit passer quelques jours avec sa mère à l'hôpital, et quand elles reviennent toutes deux à la maison, la mère d'Alissa passe l'après-midi avec elles au bord de la piscine, ce qui rend Alissa heureuse et nostalgique de ce qu'elle avait fantasmé et qui n'est pas advenu :

C'était exactement la vie de jeune maman qu'elle avait toujours imaginée : des journées oisives passées avec sa mère et la petite, au frais des orangers du patio, dans le prolongement de l'insouciante amnésie qu'avait été sa jeunesse. Ce rêve aurait pu être possible sans la trahison de sa mère, se dit-elle un peu par habitude, et sans vraie douleur, tant ce regret paraissait déjà ancien (I52).

Pendant que sa femme et son bébé séjournent à l'hôpital, Richard se rapproche de Jim. Il s'efforce en vain de sortir son ami de sa déprime et se sent fautif d'avoir lui-même été épargné grâce à la naissance d'Una. Alissa pense que comme elle, son mari regrette ses choix (I70-I72). Après une soirée chez des amis, où Alissa a le sentiment d'avoir perdu sa liberté, ses illusions, et même l'amour de Richard, et d'être définitivement incapable de prendre ses responsabilités envers son bébé, elle dépose Una dans son lit et l'abandonne. Elle roule dans la nuit, portable éteint «pour que Richard ne puisse pas la raisonner» (20I), prend une chambre d'hôtel et s'endort. Le lendemain matin, elle est soulagée de penser qu'« elle n'aurait plus jamais le courage inconscient de partir» (203). En écoutant ses messages, elle apprend que Jim a essayé d'étrangler sa femme avant de se tirer une balle dans le ventre. Arrivée chez elle, elle constate que ses voisins ont forcé la porte pour s'occuper du bébé et ont lu le billet qu'elle avait posé sur le berceau. «Alissa [le] relut, prise d'un infini regret qu'elle savait inguérissable : Désolée, je n'arriverai jamais à revenir » (205).

LImplacable Brutalité du réveil se termine ainsi par la résolution heureuse - peut-être momentanée ? - de la profonde crise traversée par la mère, qui déplore avoir délaissé son enfant et décide d'assumer ses responsabilités. Tout au long du roman, Pascale Kramer opte pour une focalisation interne sur la mère, en recourant au discours indirect, sauf en quelques rares occasions où son personnage s'exprime au discours direct et apparaît apte à analyser ce qui lui arrive. Au bord de l'épuisement, Alissa pose un diagnostic sur elle-même : " Je suis en train de faire une dépression, songea-t-elle soudain avec lucidité, presque avec soulagement » (98). Cette brusque clairvoyance sur son état ne perdure pas, et elle n'entreprend pas de demander de l'aide. Quand sa mère et Richard lui font part de leur inquiétude, sa réaction est la fuite : "Alissa dit qu'est-ce que j'y peux » (I28). Toutefois, elle arrive à formuler des reproches : "J'ai une mère qui me pousse à prendre un 
appartement bien trop petit pour élever un enfant et qui ensuite s'en fout, voilà ce que j'ai » (130). Et quand elle réalise qu'elle en veut à son enfant, elle parvient à exprimer des sentiments négatifs, articulant « tout bas et très lentement »: «Je ne t'aime pas [...]» (I36). Mais il s'avérera qu'elle ne peut pas l'abandonner.

Alissa paraît plus apte que Suzan à débrouiller la situation dans laquelle elle se trouve. Elle fournit des éclaircissements. En cela, elle s'oppose à Suzan qui, rappelons-le, ne peut, après la mort de sa fille, « rien justifier, car il n'y avait pas d'explication à tout cela. » (I69). Peut-être que dans les rares moments où Alissa s'exprime à la première personne, c'est comme si elle échappait quelque peu à l'écrivaine qui l'a créée, et qu'au final, l'expérience réussit : Pascale Kramer peut répondre à la question de savoir ce qu'on fait "lorsqu'on découvre qu'être mère est une erreur et que l'on n'assume pas ce rôle » (Rousseau 2009), grâce à l'autonomie que son personnage acquiert lorsqu'il sindividualise, et lui livre en je une analyse lucide de ses sentiments? En même temps, en romancière habile, elle livre une réponse complexe, ouverte, qui nous donne le sentiment d'avoir partagé une expérience, tout en nous laissant avec de nombreuses interrogations.

\section{Ébranler le sens du monde}

Écrire, c'est ébranler le sens du monde, y déposer une interrogation indirecte, à laquelle l'écrivain, par un dernier suspens, s'abstient de répondre. La réponse, c'est chacun de nous qui la donne, y apportant son histoire, son langage, sa liberté ; mais comme histoire, langage et liberté changent infiniment, la réponse du monde à l'écrivain est infinie : on ne cesse jamais de répondre à ce qui a été écrit hors de toute réponse : affirmés, puis mis en rivalité, puis remplacés, les sens passent, la question demeure.

Roland Barthes, Sur Racine (1963: 7)

La littérature au sens large nous ouvre à d'autres vies, que grâce à elle nous pouvons envisager, appréhender : «Bien au-delà des seuls textes de science-fiction ou de politique-fiction, ce sont tous les genres littéraires qui méritent d'apparaître comme autant de laboratoires où se formulent les possibles de notre devenir social » (Citton 2007 : I84). Pour Pascale Kramer, le roman constitue le laboratoire d'un possible - devenir mère - qui n'adviendra sans doute pas pour elle-même. Au lecteur, quelle que soit sa situation, ce laboratoire romanesque peut permettre, « un réagencement des 
sentiments et des désirs », dans la mesure où les textes fictionnels nous font « sentir et expérimenter un affect s'inscrivant en décalage avec nos réactions affectives habituelles » (I84). Cette confrontation à l'altérité a le pouvoir de nous transformer, sur un mode symbolique qui offre certains avantages :

Il semble ainsi que ce qui importe pour le rôle des dispositifs fictionnels dans l'économie psychique affective, ce ne soit pas tant le contenu de la représentation imaginaire, que le fait même du passage d'un contexte réel à un contexte fictionnel. Une des fonctions principales de la fiction sur le plan affectif résiderait ainsi dans le fait qu'elle nous permet de réorganiser les affects fantasmatiques sur un terrain ludique, de les mettre en scène, ce qui nous donne la possibilité de les expérimenter sans être submergés par eux (Schaeffer 1999: 324).

Dans Le Bateau sec et L'Implacable Brutalité du réveil, Pascale Kramer nous confronte aux doutes, aux espoirs et aux déceptions de deux mères, elle met en scène leur lassitude, leur épuisement, leur amour, et plutôt qu'une réponse, c'est la possibilité d'une expérience fantasmée qu'elle nous offre.

\section{Bibliographie}

Adamo, Ghania, J'ai des affinités avec les auteurs américains, [entretien avec Pascale Kramer], en ligne : https://www.swissinfo.ch/fre/-j-ai-des-affinités-avec-les-auteurs-américains-/7200760 (consulté le 4/II/2019).

Barthes, Roland, Sur Racine, Paris, Le Seuil, 1963.

Citton, Yves, Lire, interpréter, actualiser. Pourquoi les études littéraires ?, Paris, éd. Amsterdam, 2007.

Kramer, Pascale, Le Bateau sec, Paris, Calmann-Lévy, 1997.

-. L'Implacable Brutalité du réveil, Paris, Mercure de France, 2009, réédition Carouge-Genève, Zoé Poche, 2017.

Rousseau, Christine, «L'Implacable Brutalité du réveil: Pascale Kramer retourne à

Los Angeles », Le Monde, I9 février 2009, en ligne : https://www.lemonde. fr/livres/article/2009/o2/I9/l-implacable-brutalite-du-reveil-pascalekramer-retourne-a-los-angeles_II57705_3260.html (consulté le 4/II/20I9). Schaeffer, Jean-Marie, Pourquoi la fiction?, Paris, Seuil, 1999. 